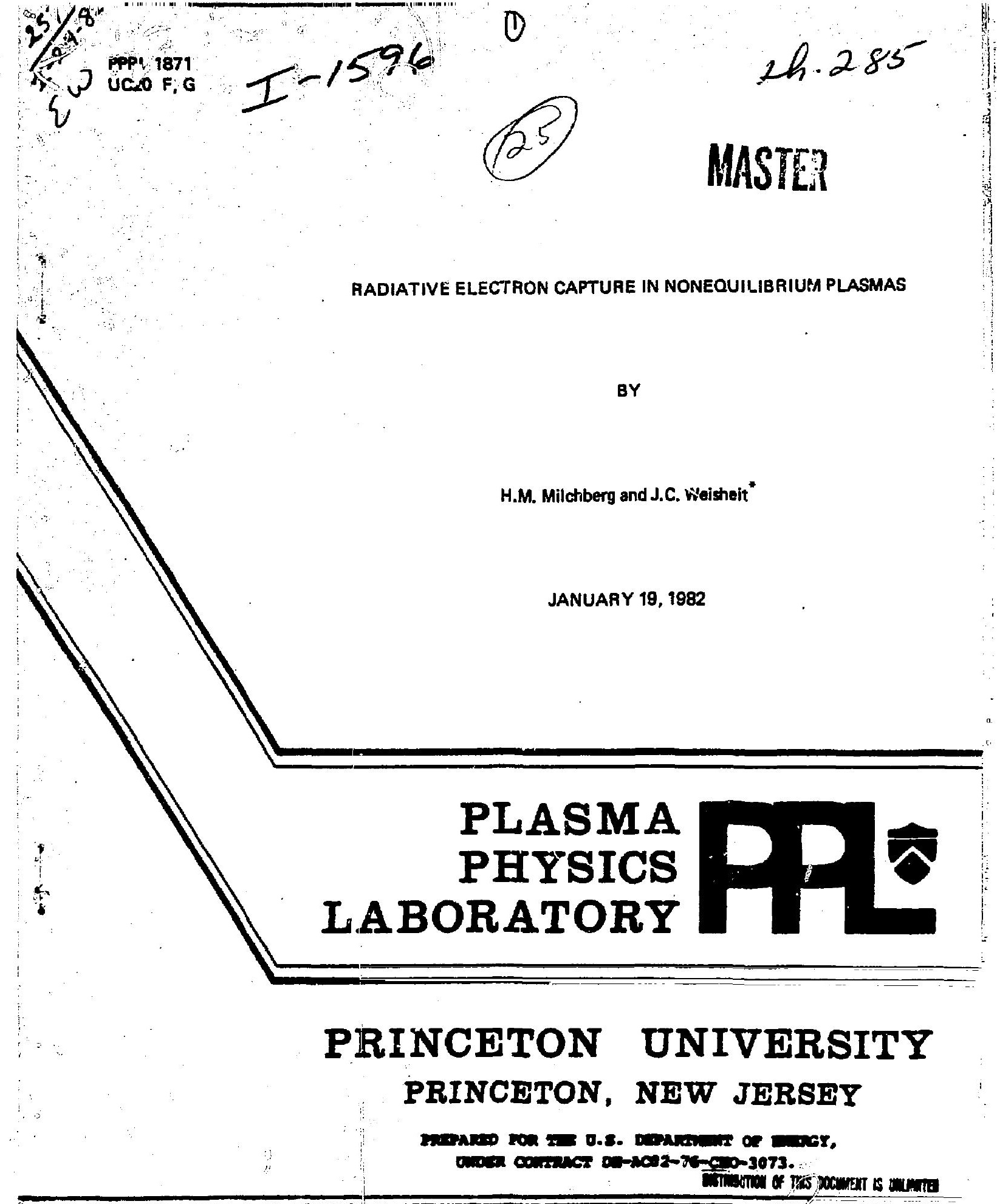




\section{Radiative Electron Capture in Nonequilibrium Plasmas}

H. M. Milchberg and J. C. Weisheit

Plasma Physics Laboratory, Princeton University

PPPI, --1871

Princeton, New Jersey 08544

DE82 011308

\section{ABSTRACT}

Formulae have been obtained for the degree of IInear polariza:ion of recombination radiation from a homogeneous plasma having an anisotropic electron velocity distribution, $f(\vec{v})$, characterized by an axis of symmetry. Polarization measurements are described which utilize tiese formulae to determine aspects of the anisotropy such as the symnetry $\therefore$ is direction and the lowest order even angular moments of $f(\vec{v})$. As a spectal case, if the plasma conforws to a distribution such as a "bi-Maxwellian with drift," one can determine the quantitles $u_{D} / T_{\|}$and $\left(1 / T_{n}-1 / T_{1}\right)$ which involve the electron dxift speed, and the "perpendicular" and "parallel" el ectron temperatures.

Also, the radiative recombination rate has been calculated for ions whose speeds are comparable to or greater than the electron thermal speed. The change in the rate is small fur therwonuclear products in fusion plasmas, but large for cosmic rays in interstellar plasma.

\section{"Pregent address: Theoretisal Phyeics Divialon, Lewrence Livermore National} Laboratory, P. O. Box 808, Livermore, Ch 94550 USA.

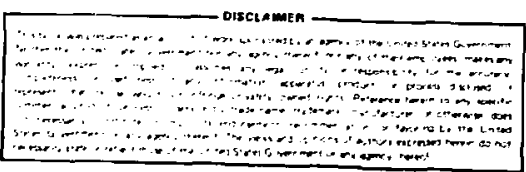

$$
\text { †ाडा }
$$




\section{INTRODUCTION}

In plasmas of sufficiently low density and high temperature, radiative electron capture, or radiative recomblnation, is an important process. The basic quantal process, which is summarized in the Appendix, is weII understood, and there exist numerous tables of thermal recombination rate coefficlents. In secs. II and III of this paper two nonequilibrium recombination phenomena are investigated. The first is the net polarization of recombination radiation that is abserved when the relative velocity distribution function is anisotropic; the second is the change in the radiative recombination rate coefficient that occurs when the ions are suprathermal with respect to the electrons. Part of our analysis is restricted to electron capture by fully stripped ions, but the extension to more complex systems is straightforward.

\section{POLARIZATION OF RECOMBINATION RADIATION}

Two often used plasma heating methods--electron cyclotron resonance radiation, 1 and lower hybrid frequency radiation ${ }^{2}$--direct energy largely into electron motions transverse to a confining magnetic fleld, and thereby give rise to anisotropies in the electron velocity distribution $f(\vec{v})$. Laser ieated plasmas are also comonly characterlzed by non-Maxwellian distributions. ${ }^{3}$ Further, solar flares evidently contain streams of accelerated electrons 4,5 and recently it has been suggested that astrophysical plasma beams carry current. 5

It has been known for some time that anlsotropies in $f(\vec{v})$ result in net linearly polarized free-free (bremsstrahlung) radiation.7,8,9 In fact, most of the anisotropies referred to above were identified by means of polarization meagurements of free-free continuum radiation. Unfortunately, 
such measurements provide limited information about $f(\vec{v})$ because a range of electron velocities contributes to free-free radiation at given frequency w. In addition, the free-free intensity $J_{2=}(\omega)$ may be less than the intensity $J_{f b}(\omega)$ of free-bound radiation from electron recombinations with highly charged ions ( $z \gg$ 1). This ls the case, for example, in spectra recorded from the Princeton Iarge Torus ${ }^{10}$ and from some laser heated plasmas. 11, 12

Except for a brief note several years ago by Paranjape, ${ }^{13}$ it seems not to have been realized that recombination emission also has net inear polarization when $f(\vec{v})$ is anisotrop1c. Moreover, in the recombination process, only electrons of specjfic relative evergy $E$ contribute to the emission at a given frequency w. For capture into a level $\Gamma$ with binding energy $\chi_{\Gamma^{\prime}}$ the nonrelativistic, center of masg energy relationship is

$$
\hbar \omega=E+\chi_{\Gamma}
$$

It is clear that, at least in principle, recombination radiation can be a more sensitive diagnostic than bremsstrahlung for plasmas in which $f(\vec{v})$ does not have a simple analytic form. This idea is developed here in some detail.

\section{A. A General Polarization Formula}

We assume that the electrons are recombining in a (homogeneous) plasma with stationary ions so that the collision energy is $E=(1 / 2) \mathrm{m}^{v^{2}}$; effects due to ion motions are discussed later. We also assume that a single spectral series dominateg the continuum radiation in the observed frequency region. Then, it follows that the number of photons of frequency w and 
polarization $\hat{e}$ emitted per unit time per unit solid angle as a result of re. mmbinations (to level $\Gamma$ ) Involving electrons of speed $v$ is

$$
\frac{d w(\hat{e}, \omega)}{d \Omega} \times \int d \Omega(\vec{v}) f(\vec{v}) v \frac{d \sigma_{\Gamma}(\hat{e}, v)}{d \Omega}
$$

where $d \Omega(\vec{v})$ is an element of solid angle in velocity space, and $d \sigma_{\Gamma} / d \Omega$ is the differential cross section for recombination to level $\Gamma$. As discussed in the Appendix, it has the general form

$$
\frac{d \sigma_{\Gamma}(\hat{e}, v)}{d \Omega}=\frac{\sigma_{\Gamma}(\hat{e}, v)}{4 \pi}\left[1+\beta_{\Gamma_{2}}(\hat{v} \cdot \hat{e} / v)\right]
$$

For recombination to the $\mathrm{k}$-shell the asymmetry parameter satisfies $\beta_{\mathrm{K}}=2$ for all ionic potentials. For higher shells, $\beta_{\Gamma}$ is given by the formula (A.7). In the Appendix it is shown that, for hydrogenic lons, the L-shell asymmetry parameter, $\beta_{L^{\prime}}$ increases from $8 / 9$ at the recombination edge to 2 as $\omega \rightarrow \infty$ (cf. Table A. 1).

Hereafter, we restrict our analysis to situations in which $f(\vec{v})$ has sylindrical symmetry, the axis of which is used to define the z-direction. Then $f(\vec{v})$ can be expanded in a series of Legendre polynomials,

$$
f(\vec{v})=\sum_{n=0}^{\infty} f_{n}(v) p_{n}(\cos \theta)
$$

and $\mathrm{Eq} \cdot(2)$ reduces to

$$
\begin{gathered}
\frac{d N}{d Q}(\hat{e}, \omega) \propto\left(2-\beta_{\Gamma}\right) K_{1}+3 \beta_{\Gamma}\left(\sin ^{2} \psi+\cos ^{2} \psi \cos ^{2} \gamma\right) K_{2} \\
+3 \beta_{\Gamma} \cos ^{2} \psi \sin ^{2} \gamma K_{3},
\end{gathered}
$$


where the angles $\psi$ and $\gamma$ are identified in Fig. 1 and where the integrals $x_{1}$ are defined as

$$
\begin{aligned}
& \mathrm{x}_{1}=2 \pi \int_{-1}^{1} \mathrm{~d}(\cos \theta) \mathrm{f}(\vec{v})=4 \pi f_{0}(v) . \\
& \mathrm{K}_{2}=2 \pi \int_{-1}^{1} \mathrm{~d}(\cos \theta) f(\vec{v})\left(\frac{1}{2} \sin ^{2} \theta\right)=4 \pi\left(\frac{1}{3} f_{0}(v)-\frac{1}{15} f_{2}(v)\right), \\
& K_{3}=2 \pi \int_{-1}^{1} d(\cos \theta) f(\vec{v})\left(\cos ^{2} \theta\right)=4 \pi\left(\frac{1}{3} f_{0}(v)+\frac{2}{15} f_{2}(v)\right) .
\end{aligned}
$$

Now consider a detector placed so as to measure radiation intensities $J_{x^{n}}(\omega)$ and $J_{z^{n}}(w)$ with polarization resolved along the $x^{\prime \prime}$ and $z^{\prime \prime}$ axes shown in F'1g. 1. Then

$$
\left.\left.J_{z^{\prime \prime}}(\omega) \propto \frac{d N(\hat{e}, \omega)}{d Q}\right|_{\phi=\rho} \quad J_{x^{n}}(\omega) \propto \frac{d N(\hat{e}, \omega)}{d \Omega}\right|_{\psi=\rho+\pi / 2} .
$$

Upon defining

$$
\Pi(\omega)=\frac{J_{z "}-J_{x "}}{J^{n}+J^{n}}
$$

as the degree of linear polarization of the recombination radiation, evaluation of Eq. (9) yields the general expression

$$
\Pi(\omega)=\frac{3 \beta_{\Gamma} \cos 2 \rho \sin ^{2} \gamma\left(k_{3}-k_{2}\right)}{2\left(2-\beta_{\Gamma}\right) k_{1}+3 \beta_{\Gamma}\left[\left(1+\cos ^{2} \gamma\right) k_{2}+\sin ^{2} k_{3}\right]}
$$




$$
=\frac{3 \beta_{\Gamma} \cos 2 \rho \sin ^{2} \gamma f_{2}}{20 f_{0}+\beta_{\Gamma} f_{2}\left(3 \sin ^{2} \gamma-2\right)} \text {. }
$$

In order for Eq. (11) to be useful, the orientation of the symmetry axis with respect to the detector's line of sight must be known. If the angles $\rho$ and $\gamma$ are unknown, they can be determined in the laboratory as follows (see Fig. 1). Let $\Pi_{0}$ denote the degree of polarization recorded when the detector is allgned with the coordinates ( $\left.x^{\prime \prime} y " z "\right) ; \Pi_{0}$ satisfies Eq. (11). Then, let $\Pi_{1}$ denote the result of a measurement made after the $\left(x^{\prime \prime} z^{\prime \prime}\right)$ axes have been rotated through an angle $\Delta_{1}$ counterclockwise about $y^{\prime \prime}$. Then $\Pi_{1}$ satisfles Eq. (11), but with $\rho+p+\Delta_{1}$. The equations for $\Pi_{0}$ and $\Pi_{1}$ can be combined to give

$$
\tan 2 \rho=\frac{\Pi_{0} \cos 2 \Delta_{1}-\Pi_{1}}{\Pi_{0} \sin 2 \Delta_{1}}
$$

This is an unambiquous determination of $p$ since $-\pi / 2 \leq \rho \leq \pi / 2$.

In order to determine the angle $\gamma$, another measurement $I_{2}$ must be made after (1) the original detector orlentation $\left(x^{\prime \prime} y^{\prime \prime} z^{\prime \prime}\right)$ has been rotated clockwise about $y^{\prime \prime}$ through the calculated angle $\rho$ to bring the $z^{\prime \prime}$ axis into the $\left(y^{\prime \prime} z\right)$ plane, and (2) this orientation has been rotated through an angle $\Delta_{2}$ clockwise about the $x$ axis. Then $\pi_{2}$ satisfies Eq. (11) but with $\gamma \rightarrow \gamma+\Delta_{2}$ and $\rho=0$. The equations for $\pi_{0}$ and $\pi_{2}$ can be combined to give

$$
\frac{\sin ^{2}\left(\gamma+\Delta_{2}\right)}{\sin ^{2} \gamma}=\frac{\Pi_{2}\left(\Pi_{0}-\cos 2 p\right)}{\Pi_{0}\left(\Pi_{2}-1\right)} .
$$


only the positive root of this quadratic equation is consistent with the combination $\Pi_{0}(\gamma=0)=0$ and $0<\Delta_{2}<\pi$. Thus

$$
\cot \gamma=-\cot \Delta_{2}+\frac{1}{\sin \Delta_{2}}\left(\frac{\Pi_{2}\left(\Pi_{0}-\cos 2 \rho\right)}{\Pi_{0}\left(\Pi_{2}-1\right)}\right)^{1 / 2} .
$$

This is an unambiguous determination of $\gamma$ since $0 \leq \gamma \leq \pi$.

\section{B. Effect of Ion Motions, Ion Charge, and Net Plagma Motion}

The effect of ion motions is to introduce some ambiguity in the inferred speeds of recombining electrons that give rise to radiation of a certain measured frequency. For electrons of a given speed $v_{e}$ one would observe a distribution of frequencies about $\omega_{0}$, the photon frequency for recombination with stationary ions. To calculate this frequency spreading, use is made of the nonrelativistic, lab frame momentum and energy conservation equations

$$
\begin{aligned}
& m_{e} \ddot{p}_{e}+m_{i} \vec{v}_{i}=m \vec{v}+h \vec{k} . \\
& \frac{1}{2} m_{e} v_{e}^{2}+\frac{1}{2} m_{1} v_{i}^{2}+\chi_{\Gamma}=\hbar \omega+\frac{1}{2} m v^{2},
\end{aligned}
$$

along with the conditions $f u r\left(m_{1} c^{2}\right), v_{1} / c, v_{e} / c$ and $m_{e} / m_{i} \ll 1$. Here $m=m_{e}+m_{i}$ is the mass of the resulting ion or atom and $\vec{v}$ is its velocity. Defining $\omega_{0}=(1 / 2) m_{e} v_{e}^{2}+x_{\Gamma^{\prime}}$ solving Eqs. (15) and (16) for $\left(\omega-\omega_{0}\right)$ and then averaging $\left(\omega-\omega_{0}\right)^{2}$ over a thermal ion velocity distribution yields for the spectral broadening, 


$$
\frac{\left\langle\left(\omega-\omega_{0}\right)^{2}\right\rangle^{1 / 2}}{\omega_{0}} \leqq \frac{1 / \sqrt{3} m_{e} v_{e} v_{i t h}}{1 / 2 m_{e} v^{2}+x_{\Gamma}}
$$

where $v_{i t h}^{2}=3 K T_{1} / m_{i}$. It is readlly seen that for sufficlently low ion temperature, high ion mass and high $\chi_{\Gamma}$, the frequency broadening is negligible, and especially so near the recombination edge $\left(v_{e} \geq 0\right)$. Moreover, this expression for the spreading is an overestimate since the slowest fons have the largest recombination cross sections, and here it has been assumed that recombination occurs with equai weight throughout the ion distribution.

High ion charges are favorable for measurements of recombination. radiation polarization. In plasmas, large $z$ ions are generally more massive, so for a given ton temperature, the thermal velocity is smaller, decreasing the frequency spreading of Eq. (17). In addition, recombination edge frequencles scale roughly as $\mathrm{z}^{2}$, further reducing this broadening and enhancing the ratio of recombination intensity to bremsstrahlung intensity. $J_{f b}(\omega) / J_{f f}(\omega) \sim \hbar \omega / K T_{e} \exp \left(h \omega / K T_{e}\right)$, near the recombination edge of a highly stripped ion.

wisen the center of mass of the plasma is not at rest with respect to the observer (e.g., a cosmic plasma), the radiation field components at the source and those at the detector are related by a Lorentz transformation. However, the net effect of the transformation is to leave the degree of polarization $\Pi$ invariant. 14

\section{c. Specific Polarization Formulae}

The coefficients $f_{0}$ and $f_{2}$ will now be evaluated for some specific velocity distributions. Tro frequently used analytic forms are a "biMaxwellian" distribution, with different temperatures $T_{\|}$and $T_{\perp}$ 
characterizing electron thermal motions parallel and perpendicular to a symuetry axis; and a Maxwellian with a net orift $\vec{u}_{D}$ which defines the symmetry axis. These two forms can be combined into a "bi-Maxwellian plus axial drift" distribution,

$$
f(\vec{v})=\left(\frac{m_{e}}{2 \pi k T_{e}}\right)^{3 / 2} \frac{T_{\|}}{T_{1}} \exp \left(-\frac{m_{e} v^{2} \sin ^{2} \theta}{2 K T_{1}}-\frac{m_{e}\left(v \cos \theta-u_{D}\right)^{2}}{2 \kappa T_{\|}}\right),
$$

and the resulting expressions for $\mathrm{f}_{0}$ and $\mathrm{f}_{2}$ are, to within the same proportionality constant,

$$
\begin{aligned}
& f_{0} \propto s(\lambda, \xi), \\
& f_{2} \propto-\frac{5}{2} s(\lambda, \xi)-\frac{15}{2} \frac{\partial s(\lambda, \xi)}{\partial \lambda} .
\end{aligned}
$$

where

$$
\lambda=\left[2 \mathrm{~m}^{\left(\hbar_{\omega} \omega-x_{\Gamma}\right)}\right]^{1 / 2} \frac{\mathrm{u}_{\mathrm{D}}}{\mathrm{KT}}, \xi=\left(\frac{\hbar \omega-\chi_{\Gamma}}{k T_{\|}}\right)\left(1-\frac{T_{\|}}{T_{1}}\right),
$$

are two dimensionless variables, and

$$
s(\lambda, \xi)=\int_{-1}^{1} d x \exp \left(-5 x^{2}+\lambda x\right)=2 \int_{n=0}^{\infty} \frac{(-\xi)^{n}}{n !} \frac{d^{2 n}}{d \lambda^{2 n}}\left(\frac{\sinh \lambda}{\lambda}\right) .
$$

In what follows, it is assumed that the orientation angles $(\rho, \gamma)$ have been determined so that it is necessary to consider only the plasma's intrinsi: polarization

$$
\tilde{\Pi}=\Pi\left(\rho=0, \quad r=\frac{\pi}{2}\right)=\frac{3 \beta_{\Gamma} f_{2}}{20 \overline{f_{0}}+\bar{\beta}_{\Gamma} \overline{f_{2}}}
$$


If $T, T_{\perp}$ and/or $\hbar \omega * \chi_{\Gamma^{\prime}}$ then $\xi$ is small and the coefficlents $f_{n}$ may he taken to first order in $\xi$, regultirg in the expression

$$
\hat{n}=\frac{3 \beta_{\Gamma}\left[a_{1}-(\xi+3) a_{2}+3 \xi a_{3}\right]}{4\left(B_{\Gamma}-2\right)\left(a_{1}-\xi a_{2}\right)+3 \beta_{\Gamma}\left[-a_{1}+(\xi-1) a_{2}+\xi a_{3}\right]},
$$

where $a_{1}=\sinh N \lambda, a_{2}=d^{2} a_{1} / d \lambda^{2}$, and $a_{3}=d^{4} a_{1} / d \lambda^{4}$.

In the limit $\xi \rightarrow 0$, Eq. (24) reduces to an expression for the degree of linear polarization due to a net drifi of plasma electrons with respect to the ions,

$$
\tilde{\pi}_{D}=\frac{3 \beta_{\Gamma}\left[3 \lambda \cosh \lambda-\left(3+\lambda^{2}\right) \sinh \lambda\right]}{2\left(\beta_{\Gamma}-2\right) \lambda^{2} \sinh \lambda+3 \beta_{\Gamma}\left[\lambda \cosh \lambda-\left(1+\lambda^{2}\right) \sinh \lambda\right]} .
$$

The asymptotic behaviors of $\tilde{\Pi}_{D}$ are

$$
\tilde{\Pi}_{D} \overbrace{\lambda \rightarrow+\infty} \frac{3 \beta_{\Gamma}}{\beta_{\Gamma}+4}, \tilde{\pi}_{D} \underset{\lambda \rightarrow 0}{\sim} \frac{\beta_{\Gamma} \lambda^{2}}{20} \text {. }
$$

The expression for $\tilde{\Pi}_{0}$ for the special case $\beta_{\Gamma}=2$ (K-shell capture) is plotter in Fig. 2.

If the drift velocity is small, then the coefficients $f_{n}$ may be taken to lowest order in $\lambda$, using the expression

$$
s(\lambda \ll 1, \xi) \approx 2 \sum_{n=0}^{\infty} \frac{(-\xi)^{n}}{n !}\left(\frac{1}{2 n+1}+\frac{\lambda^{2}}{2(2 n+3)}\right) .
$$

The limiting case $\lambda \rightarrow 0$ does not yield a closed form expression for $\tilde{\Pi}_{2 T^{\prime}}$, the degree of linear polarization of recombinatior, radiation from a biMaxwellian plasma. However, the series in 


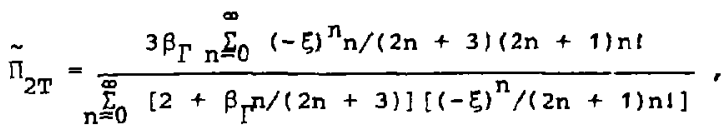

converge rapidly. The asymptotic behaviors of $\tilde{\Pi}_{2 \mathrm{~T}}$ are

$$
\tilde{\Pi}_{2 T} \underset{\xi \rightarrow \pm \infty}{ } \pm \frac{3 \beta_{\Gamma}}{\beta_{\Gamma}-\beta}, \quad \tilde{\Pi}_{2 T} \underset{\xi \rightarrow 0}{\sim}-\frac{\beta_{\Gamma} \xi}{10} .
$$

Figure 3 is a plot of $\tilde{\Pi}_{2 T}$ vs $\xi$ for $x$-ghell capture $\left(\beta_{\Gamma}=2\right)$.

\section{Comments on Polarization Measurements}

It has been assumed that a single spectral series dominates plasma emission in the observed frequency range. Often this is not the case. But, unless the characteristics of the background radiation change appreciably across the recombination edge of interest, measurements of intensity made above and below the edge can be used to subtract out the background so that $\Pi$ can be determined for that particular recombination trinsition.

In general, if polarization measurements were consistent with predictions of a particular model distribution, then the parameters $\lambda$ and/or $\xi$ could be actermined. If the measureo polarization values were not in accord with a known distribution, Eq. (11) indicates that a plot of $\Pi(\omega)$ vs $\omega$ should yield $f_{2} / f_{0}$ as a function of $w$, and hence of $v$, through Eq. (1). The lowest order even angular moments of $f(\vec{v})$ are thus determinable. Further, since the formulae which give the orientation of the symmetry axis are Independent of the form of $f(\vec{v})$, a set of polarization measurements should reveal that orlentation. A particular application of this would be the determination of magnetic field line direction.

It is interesting to compare the polarization of bremsgtrahlung with that of recombination radiation. One example is provided by the calculation 
of Raimbault and Shohet, 15. in which bremsetrahlung polartzation was determined for a bi-Maxwellian plasma with $k T_{\|}=1.5 \mathrm{KeV}$ and $k \mathrm{~T}{ }_{\perp}=15 \mathrm{KeV}$, conditions existing in their magnetic mirror device. At $h \omega=K T_{1}$, they found that the polarization of free-free radiation was about 45 percent. For these same plasma conditions, it is seen from Fig. 3 tixat, at the same frequency, the polarization of ( $K$-shell) recombination radiation exceeds this amount as long as the threshold energy $x_{\Gamma} \leq 11 \mathrm{keV}$. This inequality is satisfied for most. lons of interest.

Finally, with regard to the existence of velocity anic tropies, two irteresting astrophysical applications of polarization measurements are noted. (1) Davidson and Mccray 16 commented that there may be substantial electric currents in the jets of the bizarre galactic object ss 433 . There is strong hydrogen Balmer emission from these luminous jets, and determination of its polarization could confirm their suggestion. Hinata 17 has proposed a difficult spectroscopic observation of a combined Zeeman-Stark pattern in ت̈eI lines to determine if an energetic electron beam (instead of a thermal wave) transporis energy to the chromosphere at the beginning of a solar flare. Polarization measuremerits of the strong recombination continuum radiation (cf. Ref. 18) may also prove useful for inferring this beam's existence.

III. RADIATIVE RECOMBINATION OF SUPRATHERMAL IONS

In thermal equilibrium, plasma lons have a mean speed much less than that of the electrons, and it usually suffices to treat an electron-ton interaction as tf the lon was at rest. However, this simplification is not correct in fusion plasmas, wherein the fusion products are "born" with speeds comparable to or much greater than the electron thermal speed. The 
same problem arises whenever highly energetic lons (e.g.. stripped neutral beam particles, cosmic rays) interact with a relatively cool background plasma. In this section it is determined to what excent recombination, which favors a low relative speed $v=\left|\vec{v}_{t}-\vec{v}_{e}\right|$ of the colliding particles, is inhibited by large ion speeds.

It is assumed that the electron velocity distribution $f\left(\vec{v}_{e}\right)$ is Maxwellian at a temperature $T_{e}$ AlBo, Eq. (A.5), the cross section for bare ion recombination to ievel $n$, is approximated by Kramers" formula,

$$
\sigma_{n}(v)=\sum_{l} \sigma_{n l}(v) \cong \frac{2^{7} \pi \omega a^{2}}{3 \sqrt{3}} \frac{\left(n n \omega_{n}\right)^{3}}{\hbar\left(w\left(z n_{e} v e\right)^{2}\right.} .
$$

This approximation is valid for $\omega \leqslant 10 \omega_{1}$. Because of the assumed isotropy of the electrons, the rate coefficient for recombination to level $n$,

$$
B_{n}\left(v_{i}, T_{e}\right)=\int d^{3} v_{e} f\left(\vec{v}_{e}\right)\left|\vec{v}_{i}-\vec{v}_{e}\right| \sigma_{n}\left(\mid \vec{v}_{i}-\vec{v}_{e} \|\right),
$$

is independent of the direction of $\vec{v}_{1}$.

The angular parts of the integral Eq. (31) can be performed immediately to yield an expression in terms of the dimensionless variables

$$
x_{i}^{2}=\frac{m e^{v}{ }_{i}^{2}}{2 k T_{e}}, \quad y_{n}^{2}=\frac{\tau_{n}}{k T_{e}}
$$

namely.

$$
B_{n}\left(x_{1}, T_{e}\right)=\frac{2^{9} \pi^{2} \alpha_{0}^{2}}{3 \sqrt{3}} \frac{\left(\pi \pi \omega_{n}\right)^{3}}{(z c)^{2}}\left(\frac{1}{2 \pi e^{N T_{e}}}\right)^{3 / 2} I\left(x_{1}, y_{n}\right)
$$

The remaining integral, 


$$
\begin{aligned}
I(a, b) & =\frac{1}{a b} \int_{0}^{\infty} d x \times \exp \left(-x t^{2}\right)\left(\arctan \left[(x+a)^{2} / b^{2}\right]^{1 / 2}-\arctan \left((x-a)^{2} / b^{2}\right)^{-/ 2}\right\} \\
& =\frac{\exp \left(-a^{2}\right)}{a} \int_{0}^{\infty} d \tau \frac{\exp \left(-\tau^{2}\right) \sinh (2 a \tau)}{\left(\tau^{2}+b^{2}\right)},
\end{aligned}
$$

can be evaluated term by term:

$$
I(a, b)=\exp \left(-a^{2}\right)\left(\frac{\sin (2 a b)}{2 a b} I(0, b)+\sum_{m=1}^{\infty} \frac{\left(-4 a^{2} b^{2}\right)^{m}}{(2 m+1) !} \sum_{p=1}^{m} \frac{(p-1) !}{\left(-b^{2}\right)^{p}}\right) .
$$

In this expression

$$
I(\bar{u}, b) \equiv \lim _{a \rightarrow 0} I(a, b)=\exp \left(b^{2}\right) E_{1}\left(b^{2}\right)
$$

where $E_{1}$ is the first exponential integral functior.

Two limiting forms of $\mathrm{Eg}$. (35) are of interest. When $b \ll 1$, $E_{1}\left(b^{2}\right) \sim-\ln b^{2}$ so that

$$
I(a, b<1) \cong \exp \left(-a^{2}\right) \ln \left(1 / b^{2}\right)
$$

When $b$ is large and the $p=1$ term dominates,

$$
\begin{aligned}
I(a, b) \mid 1) & \approx \exp \left(-a^{2}\right)\left[\frac{\sin (2 a b)}{2 a b} I(0, b)+\frac{1}{b^{2}}\left(1-\frac{\sin (2 a b)}{2 a b}\right)\right] \\
& \approx \exp \left(-a^{2}\right) / b^{2}
\end{aligned}
$$

since $I(0, b \gg 1)=b^{-2}$. Also, it is easily verified that in the 
Iimit $v_{i} \rightarrow 0$, the usual hydrogenic formula is regained:

$$
B_{n}\left(0, T_{e}\right)=\frac{2^{6} \alpha^{3} a_{0}^{2} c n}{3}\left(\frac{\hbar \omega_{n}}{k T_{e}}\right)^{2}\left(\frac{i \kappa T}{e}\right)^{1 / 2} \exp \left(\frac{\hbar \omega_{n}}{K T_{e}}\right) E_{1}\left(\frac{\hbar \omega_{n}}{K T_{e}}\right) .
$$

For a particles produced in D-T fusion plasmas, the parameter $y_{n} \ll 1$ for all $\mathrm{n}$. For $(1 / 2) \mathrm{m}_{\alpha} \mathrm{v}_{\alpha}^{2}=4 \mathrm{MeV}, x_{\alpha} \approx(0.5 \mathrm{KeV} / \mathrm{KT})^{1 / 2}<1$ and a satisfactory approximation is

$$
\frac{B_{n}\left(x_{\alpha^{\prime}} T_{e}\right)}{B_{n}\left(0, T_{e}\right)}=\exp \left(-x_{\alpha}^{2}\right)\left(1-\frac{2 x_{\alpha}^{2}}{3 \ln \left(y_{n}^{2}\right)}\right)
$$

It is interesting to note that this $x_{\alpha}$ dependence is the same as that for the ratio of the number of electrons in each case $\left(v_{\alpha} \neq 0\right.$ and $\left.v_{\alpha}=0\right)$ which, relative to the lon, have speeds smaller than some maximum value $\left.\hat{v} \cong\left(2 \times T e^{/ m_{e}}\right)^{1 / 2 / 1 n(K T} e^{/ n \omega_{n}}\right)$ for efficient electron capture. Because $x_{\alpha}$ typically is $\leq 0.1$, the $\alpha$-particle's speed is not high enough to reduce its recombination rate coefficient very much.

On the other hand, the rate coefficlent for free electron capture by a cosmic ray particle of energy $E_{C R}$ traversing intersteliar plasma $\left(\mathrm{kT}_{\mathrm{e}} \geq 1 \mathrm{eV}\right)$ is reduced significantly by 1 ts motion. In this case, relevant to low energy cosmic rays, the parameter $y_{n}^{2}$ is large for the lower states $(n \leq 4)$ and $x_{C R}^{2} \cong 5 E_{C R}($ MeV/amu) $>1.21$ Therefore, from Eqs. (38) and (39) it follows that

$$
\frac{B_{n}\left(x_{C R^{\prime}} T_{e}\right)}{B_{n}\left(0, T_{e}\right)}=\exp \left(-x_{C R}^{2}\right) \ll 1 .
$$

Here, the $x_{\alpha}$ dependence corresponds simply to the Boltzmann factor for electrons having speeds $v_{e} \tilde{z} v_{\mathrm{CR}^{\circ}}$. 


\section{ACKNOWLEDGMENTS}

The topic of Sec. II arose in conversations one of the authors ( $J$. C. W.) had with Dr. Eric Silver. Both he and Dr. Doug Post are thanked for useful discussions.

This research was supported by the United states Department of Energy under Contracts DE-ACO2-76-CH03073 and $\mathrm{w}-7405-E N G-48$. H. Milchberg acknowledges the support of the Natural Sciences and Engineering Research Council of Canada. 
APPENDIX: THE RECOMBINATION CROSS SECTION

In the dipole approximation, the differential cross section for an electron and an ton with reduced mass $\mu$ and relative momentum fia $=\vec{q} \vec{v}$ to recombine and emit a photon of polarization $\hat{e}$ and momentum $\vec{i} \vec{k}$ within the differential solid angle $d \Omega$ about $\vec{k}$ is

$$
d v(\hat{e}, q)=\frac{k^{3}}{2 \pi \vec{r}} \mid\left\langle\bar{e}, \text { ion }|\hat{e} \cdot \vec{a}| a t o m>\left.\right|^{2} d \Omega .\right.
$$

where $d$ is the instantaneous dipole moment of the electron-ion system, and where the continuum wave function is normalized to unit flux.

When the bound state is specified by hydrogenlc quantum numbers, e.g., |atom $\rangle=|\mathrm{n} \ell \mathrm{m}\rangle$ and the contiriuum state is expanded in partial waves,

$$
\mid \vec{e}, \text { ion })=\frac{4 \pi}{q}\left(\frac{\pi \mu}{2 \pi q}\right)^{1 / 2} \sum_{\lambda, \nu} i^{\lambda} \exp \left(i \eta_{\lambda}\right) \mathbf{Y}_{\lambda \nu}^{*}(\dot{q}) Y_{\lambda \nu}(\hat{r}) R_{q \lambda}(x) .
$$

Equation (A.1) becomes

$$
\begin{aligned}
& d \sigma_{n \ell}(\hat{e}, q)=\left.\left(\frac{16 \pi^{3} e^{2} k^{3} \mu}{m^{2} q^{3}}\right) \int_{m}\right|_{\lambda=\ell \pm 1}(-i)^{\lambda} \exp \left(-i, \eta_{\lambda}\right)[\max (l, \lambda)]^{1 / 2} \rho_{\lambda} \\
& \times\left.\sum_{V M} Y_{\lambda \nu}(\hat{q}) Y_{q M}(\hat{e})\left({ }_{\nu M m}^{\lambda}\right)\right|^{2} d Q .
\end{aligned}
$$

In this equation $\left(\begin{array}{lll}l_{1} & l_{2} & l_{3}\end{array}\right)$ is a $3-j$ symbol, $y_{\lambda \nu}$ is a spherical harmonic, $\rho_{\lambda}=\left\langle q \lambda|r| n \oslash\right.$ is a ${ }^{m}{ }^{m} d^{2}{ }^{m} a^{3}$ matrix element, and

$$
\eta_{\lambda}=\arg \Gamma\left(1+\lambda-1 \frac{\mathbf{z a c}}{v}\right),
$$

Is the coulomb phase shift. Note that the cross section has been oummed 
with respect to degenerate atomic stateg having different asimuthal quantum numbers $m$. Integration with respect to solid angle yields the cross section for recombination into level $n l$, with the emission of a photon of specified Erequency and polarization,

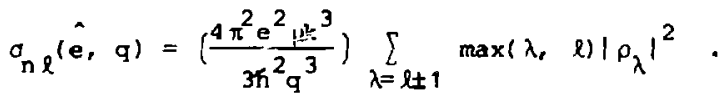

The total cross section $\sigma_{n l}(q)$, which involves a summation with respect to polarization states, is simply twice this expression.

Cooper and Zare 19 showed that the equation for the differential cross section Eq. (A.3) can be simplified to yield

$$
d \sigma_{n \ell}(\hat{e}, q)=\sigma_{n \ell}(\hat{e}, q)\left[1+\beta_{n \ell} P_{2}(\vec{q} \cdot \hat{e} / q)\right] d \Omega / 4 \pi \text {. }
$$

where $\mathrm{P}_{2}(x)$ is the aecond order Legendre polynomial and where the asymetr: parameter

$$
\beta_{n \ell}=\frac{l(l-1) \rho_{l-1}^{2}+(l+1)(l+2) \rho_{l+1}^{2}-6 l(l+1) \rho_{l-1} \rho_{l+1} \cos \left(\eta_{l+1} \eta_{l-1}\right)}{(2 \ell+1)\left[l \rho_{l-1}^{2}+(l+1) \rho_{l+1}^{2}\right]},
$$

is defined in terms of continuum phase shifts $\eta$ and radial matrix elements p. In general, $\beta_{n \ell}$ varles between -1 and 2 , but for $s$ states $(l=0) \quad \beta_{n s}=2$, independent of $\eta, \rho$, and $q$. Thus for the $k$-shell

$$
\frac{d \sigma_{x}}{d \Omega}(\hat{e}, q)=\frac{\sigma q_{1 s}(\hat{e}, q)}{4 \pi}\left[1+2 P_{2}(\vec{q} \cdot \hat{e} / q)\right],
$$

while for the L-shell 


$$
\begin{aligned}
\frac{d \sigma_{L}(\hat{e}, q)}{d \Omega}= & \frac{\sigma_{2 s}(\hat{e}, q)}{4 \pi}\left[1+2 p_{2}(\vec{q} \cdot \hat{e} / q)\right] \\
& +\frac{\sigma_{2 p}(\hat{e}, q)}{4 \pi}\left[1+\beta_{2 p} P_{2}(\vec{q} \cdot \hat{e} / q)\right] \\
= & \frac{\sigma_{L}(\hat{e}, q)}{4 \pi}\left[1+\beta_{L} p_{2}(\vec{q} \cdot \hat{e} / q)\right] .
\end{aligned}
$$

The total L-shell recombination cross section is $\sigma_{I}=\sigma_{2 s}+\sigma_{2 p^{\prime}}$ and the effective asymmetry parameter for the L-shell is

$$
\beta_{L}=2+\left(\beta_{2 p}-2\right) \frac{\sigma_{2 p}}{\sigma_{L}}
$$

In order to calculate $\beta_{2 p}$ we use the general rojult

$$
\eta_{l+1}-\eta_{l-1}=\arctan \left(\frac{(2 \ell+1) \tau_{n}}{\tau_{n}^{2}-\ell(\ell+1)}\right),
$$

where the parameter

$$
\tau_{n}=\frac{z a c}{v}=n\left(\frac{\omega_{n}}{\omega-\omega_{n}}\right)^{1 / 2}\left(\frac{\mu}{m_{e}}\right)
$$

is defined in terms of the photon energy $\hbar \omega$ and the binding energy $x_{n}$ of the $n^{\text {th }}$ level. In addition, Burgess' formulae 20 for the radial matrix elements $p_{\lambda}$ are used to obtain for the $2 p$ state

$$
\frac{\rho_{2}}{\rho_{0}}=2\left(\frac{\omega+3 w_{2}}{\omega}\right)^{1 / 2},
$$

and 


$$
\frac{\sigma_{2 p}}{\sigma_{L}}=\frac{\left(w_{2} / \omega\right)\left(3+8 \omega_{2} / \omega\right)}{\left(1+3 \omega_{2} / \omega\right)+\left(w_{2} / \omega\right)\left(3+8 \omega_{2} / \omega\right)} .
$$

Altogethe:, Eqs. (A.7) and $(A .10)$ - (A.14) yield the values of $\beta_{2 p}$ and $\beta_{L}$ Iisted in Table A.I.

For simplicity, only $K$ and $L$-shell capture of an electron by a bare nucleus has been consiliered. More complicated ions and higher shells can be treated by generalization of the equations given here. 
TAELE A. 1.

HYDROGENIC ASYMMETRY PARAMETERS

\begin{tabular}{|c|c|c|}
\hline$\omega / \omega_{2}$ & $\beta_{2 p}$ & $\beta_{L}$ \\
\hline 1.00 & 0.48485 & 0.88889 \\
\hline 1.02 & 0.49186 & $0.8997 \%$ \\
\hline 1.04 & 0.49880 & 0.91047 \\
\hline 1.06 & 0.50566 & $0.9210 \%$ \\
\hline 1.08 & 0.51246 & 0.93138 \\
\hline 1.10 & 0.51917 & $0.94161)$ \\
\hline 1.15 & 0.53566 & 0.96646 \\
\hline 1.20 & 0.55172 & 0.99038 \\
\hline 1.25 & 0.56738 & 1.0134 \\
\hline 1.30 & 0.58263 & 1.0356 \\
\hline 1.40 & 0.61202 & 1.0777 \\
\hline 1.50 & 0.64000 & 1.1169 \\
\hline 1.75 & 0.70440 & 1.2039 \\
\hline 2.00 & 0.76190 & 1.2778 \\
\hline 2.50 & 0.86022 & 1.3960 \\
\hline 2.90 & 0.92615 & 1.4696 \\
\hline 3.00 & 0.94118 & 1.4857 \\
\hline 3.10 & 0.92486 & 1.4863 \\
\hline 3.50 & 0.86486 & 1.4909 \\
\hline 4.00 & 0.80000 & 1.5000 \\
\hline
\end{tabular}




\section{REFERENCES}

1D. G. S. Greene and J. L. Shohet, Plasma Phys. 15, 5 (1973).

${ }^{2}$ S. B. Litvin and V. T. Tikhonchuk, Sov. J. Plasma Phys. 6, 183 (1980).

${ }^{3}$ G. Beikefi, C. Deutsch, and B. Yaakob1, in Principles of Laser plasmas, ed.

G. Bekefi, Wiley-Interscience, New York (1976), pp 596-602.

${ }^{4}$ G. A. Dulk, D. B. Melrose, and S. F. Smerd, Proc. Astron. Soc. Australia 3 , $243(1978)$.

${ }^{5}$ A. G. Emslie and J. C. Brown, Astrophys. J. 237, 1015 (1980).

${ }^{6}$ G. Benford, Mon. Not. Roy. Astron. Soc. (London) 183, 29 (1978).

7R. L. Gluckstern and M. H. Hull, Phys. Rev. 90, 1030 (1953),

A. A. Korchak, Sov. Phys. Doklady 12, 192 (1967).

${ }^{9}$ K. Eidmann, Plasma Phys. 17, 121 (:975).

10K. Brau, S. Von Goeler, M. Bitter, R. D. Cowan, D. Eames, K. Hill, N. Sauthoff, E. Silver, and W. Stodiek, Phys. Rev. A. 22 (6), 2769 (1980).

11M. H. Key, J. G. Lunney, J. M. Ward, R. G. Evans, and P. T. Rumsby, J. Phys. B. 12 , L213 (1979).

${ }^{12}$ G. Charatis, D. C. Slater, F. J. Mayer, J. A. Tarvin, G. E. Busch, D. L. Matthews, L. Koppel, in Low Energy X-ray Diagnostics - 1981, ed. D. Attwood, B. Henke, A.I.P., New York (1981).

${ }^{13}$ v. Paranjape, Proc. Roy. Soc. 69B, 765 (1956).

${ }^{14}$ L. D. Landau and E. M. Lifschitz, Classical Theory of Fie:ds, 3rd. ed., Pergamon Press, Oxford (1971), pp. 119-123.

${ }^{15}$ P. A. Rairabault and J. L. Shohet, Phys. Fluids 15, 1477 (1972).

${ }^{16}$ K. Davidson and R. McRay, Astrophys. J. 241, 1082 (1980).

${ }^{17}$ s. Hinata, Astrophys. J. 234, 363 (1979).

${ }^{18} \mathrm{~J}$. T. Jeffries and F. Q. Orrall, Astrophys. J. 133, 946 (1961). 
${ }^{19} \mathrm{~J}$. Cooper and R. N. Zare, in Atomic Coll1sion Processes, ed.

S. Geltman, K. T. Manthappa, w. E. Brittin, Gordon and Breach, New York (1969) pp. 317-335.

20 A. Burgess, Mem. Roy. Astron. Soc. (London) 69, 1 (1964).

2 'v. L. Ginzburg, Elementary Processes for Cosmic Ray Astrophysics, Gordon and Freach, New York (1969), Section I.1. 


\section{Figure Captions}

Fig. 1. Geometry of observation of recombination radiation. The ine of sight lies in the yz plane.

Fig. 2. Degre of linear polarization vs. $\lambda$ for the drift case.

Fig. 3. Degree of linear polarization vs. $\xi$ for the two temperature case. 
\#81T0371

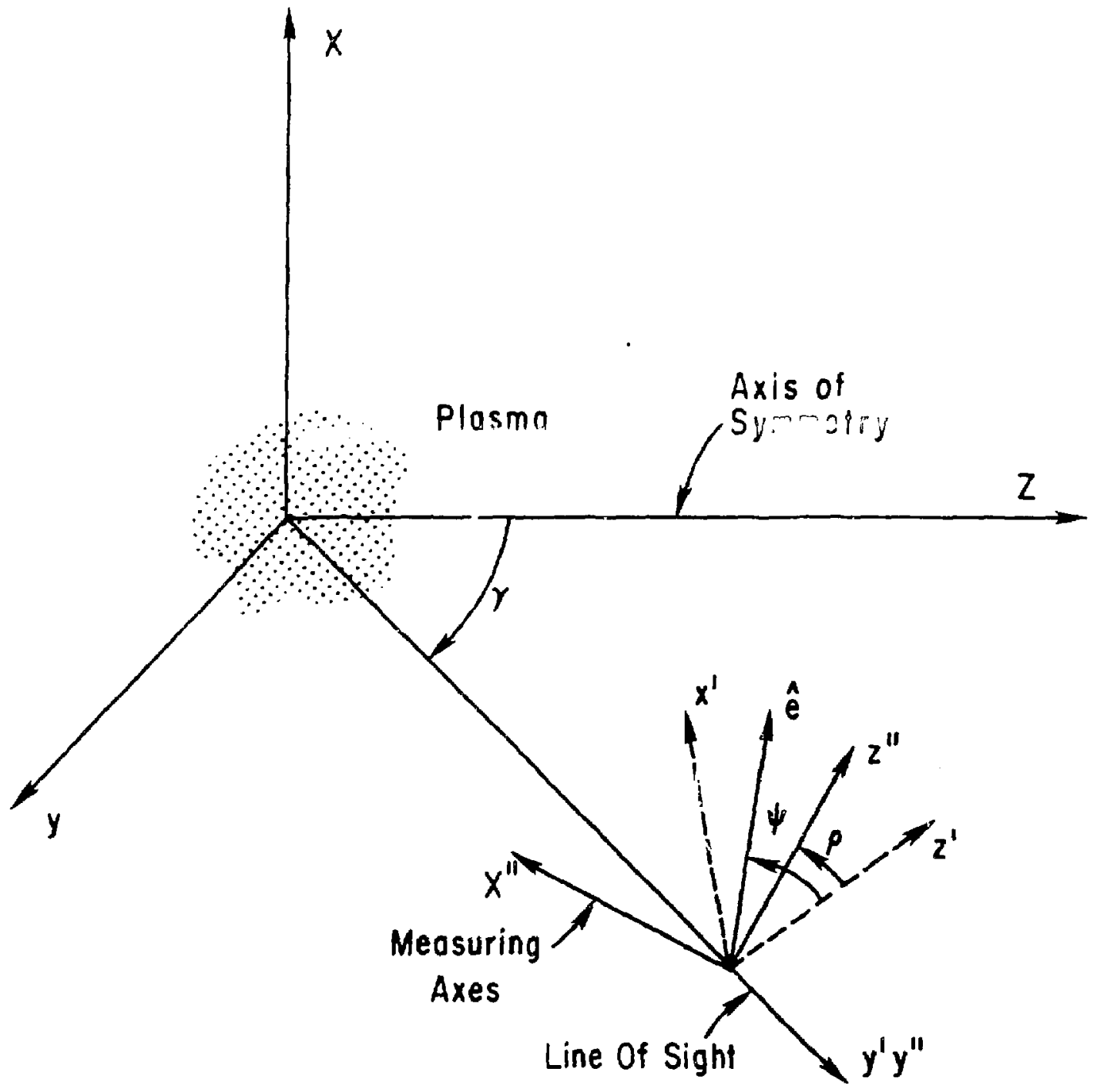

Fig. I 
\# 81 T0373

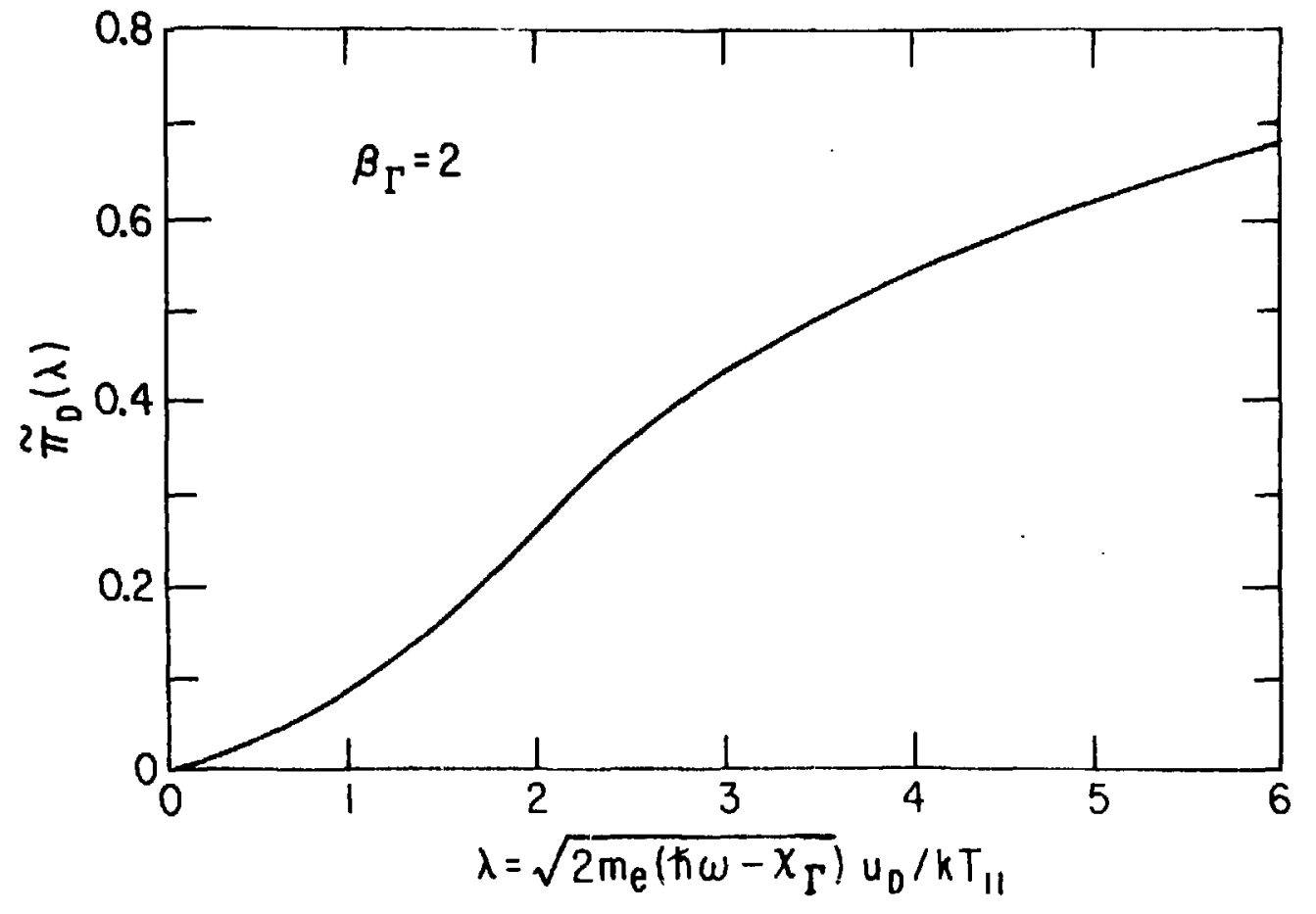


\#81т0372

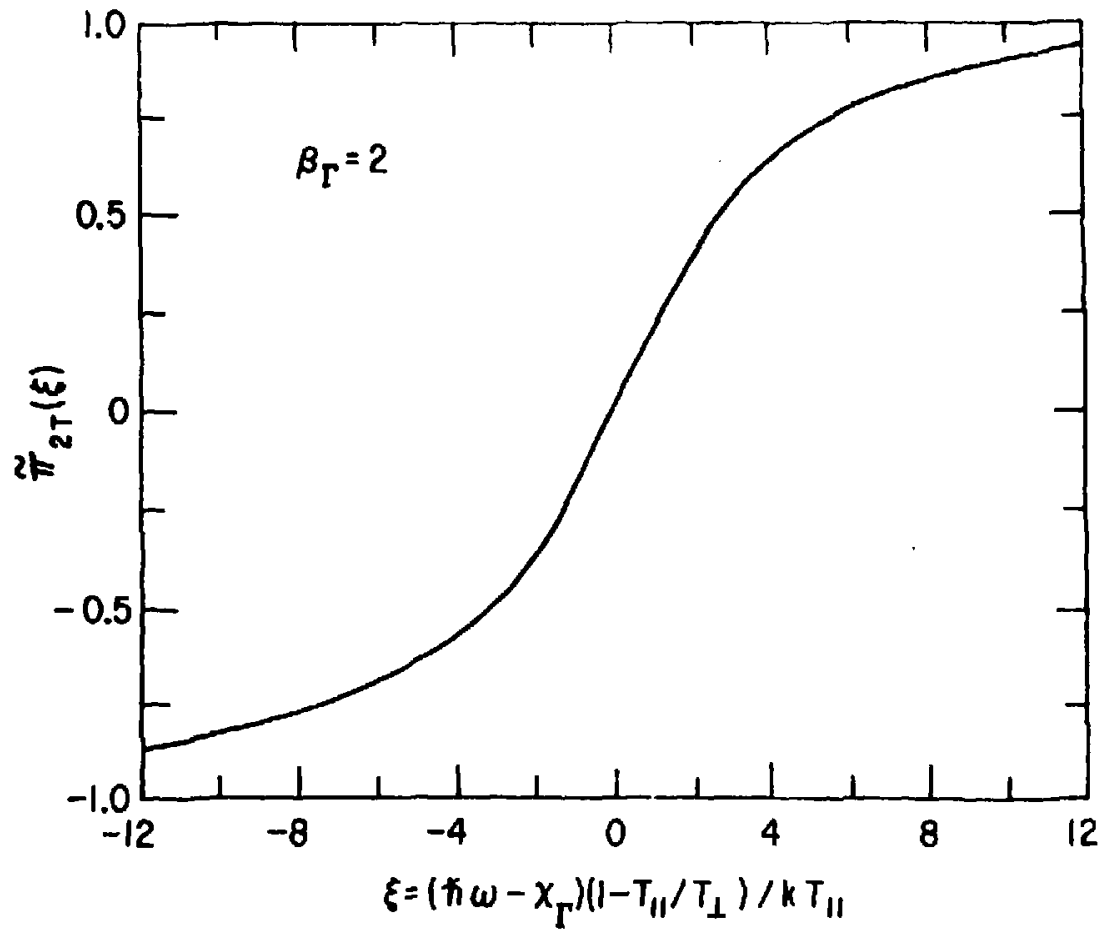

ria.? 